\title{
Efeito do Genótipo sobre as Medidas Objetivas e Subjetivas da Carcaça de Cordeiros Terminados em Confinamento ${ }^{1}$
}

\author{
Edson Ramos de Siqueira ${ }^{2}$, Simone Fernandes ${ }^{3}$
}

\begin{abstract}
RESUMO - Este experimento foi realizado para comparar as medidas objetivas e subjetivas da carcaça de 36 cordeiros, 18 da raça Corriedale e 18 mestiços (F1) Ile de France x Corriedale, abatidos com 30 a 32 kg PV. Os cordeiros foram desmamados aos 60 dias de idade, com 10 a $12 \mathrm{~kg} \mathrm{PV}$, e terminados em regime de confinamento total, no qual dispunham, à vontade, de uma ração com 15,0\% PB e 70,0\% NDT. Terminada a manipulação pós-abate e o resfriamento por 24 horas a $4{ }^{\circ} \mathrm{C}$, avaliações objetivas e subjetivas nas carcaças foram realizadas. Houve diferenças entre os grupos genéticos estudados somente quanto ao comprimento externo e interno da carcaça, tendo os cordeiros puros apresentado maiores valores para essas medidas. Entretanto, por meio da média geral constatada para a área do longissimus dorsi, foi observado que os dois grupos genéticos apresentaram a mesma quantidade de músculo na carcaça. As carcaças dos cordeiros mestiços (IF x C) foram consideradas de melhor conformação e apresentaram a mesma quantidade de gordura de cobertura que as de inferior conformação. Os genótipos estudados, de maneira geral, foram capazes de atender dois importantes requisitos: a exigência do mercado consumidor por carcaças que não apresentem excesso de tecido adiposo e a necessidade de pequena cobertura de gordura, para que a qualidade da carne não seja prejudicada.
\end{abstract}

Palavras- chave: carcaça, carne ovina, cordeiro, cruzamento

\section{Effect of Objective and Subjective Measurements of Carcasses from Lambs Finished in Feedlot}

\begin{abstract}
This experiment was carried out to compare the objective and subjective measurements of carcasses from 18 Corriedale and 18 Ile de France x Corriedale lambs, slaughtered at 30 to $32 \mathrm{~kg} \mathrm{LW}$. The lambs were weaned at 60 days of age, with 10 to $12 \mathrm{~kg} \mathrm{LW,}$ finished in feedlot, and were full fed a diet with $15,0 \% \mathrm{CP}$ and 70,0\% TDN. After post-slaughter manipulation and cooled for 24 hours at $4{ }^{\circ} \mathrm{C}$, the objective and subjective carcass evaluations were made. There were differences between the studied genotype groups only for carcass external and internal length. The straightbreds lambs presented higher values for those measurements. However, by the overall mean observed for the longissimus dorsi area, the genotype groups presented the same amount of muscle in the carcass. The crossbred lamb carcass (IF x C) were considered to have the best conformation and presented the same subcutaneous fat cover of that of pour conformation. The studied genotype groups, in overall, were capable to meet two important demand of meat market requirements for lean carcass but with small subcutaneous fat cover to conserve the meat quality.
\end{abstract}

Key Words: carcass, lamb meat, lamb, crossbreeding

\section{Introdução}

O estudo dos aspectos qualitativos das carcaças ovinas produzidas nas distintas regiões criatórias revestese de capital importância, haja vista as peculiaridades dos sistemas de produção e dos genótipos utilizados.

EL KARIN et al. (1988) relatam que o efeito do genótipo é evidente, quando se usam algumas medidas na predição da composição da carcaça. O uso de melhor alimentação e cruzamentos industriais pode causar sensíveis modificações em características como comprimento da carcaça e da perna e peso da carcaça (CARVALHO et al., 1980).
SIERRA e BOCCARD (1970) verificaram que cordeiros puros da raça Rasa Aragonesa apresentaram carcaças contendo planos musculares menos desenvolvidos, com pernas longas e pouco arredondadas - características associadas ao maior comprimento interno da carcaça, ao maior comprimento da perna e à menor largura do tórax, quando comparados aos mestiços Texel $x$ Rasa Aragonesa, que revelaram carcaças mais curtas e com massas musculares mais desenvolvidas.

Resultados citados por PALSSON (1939) mostraram que a profundidade do músculo longissimus dorsi (medida B), determinada na penúltima costela, é medida indicadora da musculatura total da carcaça,

\footnotetext{
${ }^{1}$ Projeto financiado pela FAPESP.

2 Professor Adjunto do DPEA - FMVZ/UNESP - 18618-000, Botucatu/ SP. Fax: (0XX14) 8207180. E. mail: e.r.siqueira@fca.unesp.br

3 Zootecnista - DPEA - FMVZ/UNESP - 18618-000, Botucatu/ SP.
} 
ao passo que a espessura da gordura de cobertura apresenta alta correlação com a gordura subcutânea total da carcaça.

A área do músculo longissimus dorsi ou área de olho de lombo é considerada medida representativa da quantidade e distribuição das massas musculares, assim como da qualidade da carcaça (BONIFACINO et al., 1979).

Em cruzamento da raça Ile de France com Merino da Turquia, também observou-se maior valor para área de olho de lombo nas carcaças dos animais mestiços $\left(23,5 \mathrm{~cm}^{2}\right)$, comparativamente aos puros Merino $\left(20,0 \mathrm{~cm}^{2}\right)$, todos abatidos aos 105 dias de idade (AKÇAPINAR, 1976).

Segundo SIERRA et al. (1992), o genótipo e o sistema de alimentação podem variar de forma significativa a relação entre a conformação visual e a composição física da carcaça. A conformação está fundamentalmente influenciada pela base genética, sendo que as raças bem conformadas, de clara aptidão para a produção de carne, transmitem à sua descendência boa morfologia, enquanto as raças rústicas apresentam, em geral, carcaças estreitas (Kempster, 1980, citado por SAÑUDO e SIERRA, 1986).

A avaliação objetiva da conformação pode ser efetuada mediante uma série de medidas da carcaça, e algumas podem originar índices de compacidade (OSÓRIO 1992), para dar valor à distribuição da carne e da gordura na carcaça (TWAITES et al. 1964).

Segundo THORNTON et al. (1974), a distribuição da gordura é função de peso vivo, peso da carcaça, sexo, tipo de alimentação e raça. Já THOMPSON e BUTTERFIELD (1987) enfatizam as diferenças verificadas entre os genótipos.

O excesso de gordura é fator limitante na produção de cordeiros mais pesados, sendo necessária a identificação de raças e cruzamentos capazes de produzir carcaças mais desejáveis, sobretudo quando os animais são alimentados para atingirem pesos elevados (LAMBUTH et al. 1970).

O objetivo deste trabalho foi comparar cordeiros da raça Corriedale e mestiços Ile de France $x$ Corriedale, recriados e terminados em confinamento, quanto às medidas objetivas e subjetivas da carcaça.

\section{Material e Métodos}

O presente experimento foi conduzido na Faculdade de Medicina Veterinária e Zootecnia da UNESP, Campus de Botucatu. Utilizaram-se 36 cordeiros inteiros, oriundos de dois genótipos, sendo 18 da raça Corriedale e 18 mestiços (F1) Ile de France x
Corriedale. Todos os animais eram da mesma origem e permaneceram com as mães, exclusivamente em pastagem de Cynodon dactylon, cv. coast-cross, até a desmama efetuada aos 60 dias de idade média, quando tinham de 10 a $12 \mathrm{~kg}$ de peso vivo.

A terminação dos cordeiros foi realizada em regime de confinamento total, no qual dispunham de uma ração com $15 \%$ de proteína bruta (PB) e $70 \%$ de nutrientes digestíveis totais (NDT), ad libitum, com a seguinte composição: $35,00 \%$ de feno de coast-cross, $22,55 \%$ de milho moído, 21,35\% de farelo de algodão, 20,00\% de esterco de galinha, $0,60 \%$ de calcário, $0,20 \%$ de premix vitamínico-mineral e $0,20 \%$ de sal iodado.

Os animais foram abatidos quando atingiam peso entre 30 e 32 kg. Registrou-se, anteriormente à degola, a condição corporal, avaliada subjetivamente mediante palpação da região lombar do animal, com atribuição de nota de 1 a 5 ( 1 para a pior, 5 para a melhor).

Terminada a manipulação pós-abate, as carcaças foram submetidas a resfriamento por 24 horas, a $4^{\circ} \mathrm{C}$ de temperatura.

Ao final deste período, as carcaças foram mantidas com as articulações tarso metatarsianas distanciadas em $17 \mathrm{~cm}$, por meio de gancho próprio, tomando-se as medidas abaixo (SAÑUDO e SIERRA, 1986):

Comprimento da perna (CP): distância entre o períneo e o bordo anterior da superfície articular tarso metatarsiana, tomada com fita métrica;

Comprimento interno da carcaça (CIC): distância máxima entre o bordo anterior da sínfise ísquiopubiana e o bordo anterior da primeira costela em seu ponto médio, tomada com fita métrica;

Comprimento externo da carcaça (CEC): é a distância entre a base da cauda e a base do pescoço, medida com fita métrica;

Largura da garupa (LG): largura máxima entre os trocânteres de ambos os fêmures; tomada com compasso;

Perímetro da garupa (PG): é o perímetro desta região anatômica, tomando-se como referência os trocânteres de ambos os fêmures;

Largura do tórax (LT): é a largura máxima da carcaça no nível das costelas, tomada com compasso; e

Profundidade do tórax (PT): é a distância máxima entre o esterno e o dorso da carcaça, tomada com compasso.

Foram calculados os índices de compacidade da carcaça (peso da carcaça fria dividido pelo comprimento interno da carcaça) e de compacidade da perna (largura da garupa dividida pelo comprimento da perna). 
308 Rev. bras. zootec.

As carcaças foram avaliadas subjetivamente segundo metodologia citada por COLOMER ROCHER (1986), considerando-se as seguintes variáveis:

Grau de gordura (GG): determinado mediante apreciação visual, utilizando-se uma escala de 5 pontos, sendo o valor 1 para a excessivamente magra e 5, para a excessivamente gorda; e

Grau de conformação (GC): avaliação visual da carcaça, considerando-a como um todo, e levando-se em consideração as diferentes regiões anatômicas (perna, garupa, lombos e espádua) e a espessura de seus planos musculares e adiposos, em relação ao tamanho do esqueleto, que a suporta escala de 1 a 5 , sendo o valor 1 atribuído à conformação muito pobre e 5 para a excelente.

A área transversal do músculo longissimus dorsi (entre a última vértebra torácica e a primeira lombar, no corte denominado lombo) foi determinada traçando-se seu perfil em papel vegetal, para posterior mensuração com planímetro.

Sobre a superfície do longissimus dorsi, tomaram-se as medidas abaixo citadas:

Medida A ou largura máxima do músculo longissimus dorsi, perpendicular ao eixo ou medida $\mathrm{B}$, tomada com régua;

Medida B ou profundidade do músculo longissimus dorsi: é a profundidade máxima do mesmo, tomada com régua;

Medida $\mathrm{C}$ ou espessura de gordura sobre o músculo longissimus dorsi: é a espessura da gordura de cobertura sobre a secção transversal do referido músculo, à continuação do eixo $\mathrm{B}$, tomada com paquímetro; e

Medida J: é a maior espessura da gordura de cobertura, tomada com paquímetro, no perfil do corte denominado lombo, no qual foram obtidas as medidas anteriores.

A comparação entre os dois genótipos foi efetuada, segundo método estatístico de ZAR (1984), utilizando-se:

a) para as variáveis quantitativas, o Teste $t$ para duas amostras independentes, com a determinação da média como medida de tendência central;

b) para as variáveis semi-quantitativas (notas), a Prova Não-Paramétrica de Mann-Whittney citada por ZAR (1984), com a determinação da mediana como medida de tendência central.

Foram determinados os coeficientes de correlação linear entre pares de variáveis.

\section{Resultados e Discussão}

As médias e os desvios-padrão, para as medidas citadas, encontram-se na Tabela 1 . Houve diferenças significativas entre os grupos genéticos estudados, somente, no que se refere ao comprimento externo e interno da carcaça. Estes resultados concordam com os de SIERRA e BOCCARD (1970) e HUIDOBRO e JURADO (1989), os quais constataram que os cordeiros puros apresentaram carcaças mais longas que os mestiços, com pesos de carcaça similares aos do presente experimento $(11 \mathrm{~kg})$. Entretanto, ao se verificarem as demais medidas, principalmente comprimento da perna, largura e perímetro da garupa, constatou-se que os cordeiros puros foram similares aos mestiços, caracterizando-se por possuírem carcaças com perfil subconvexo, o qual configura planos musculares adequados.

A similaridade dos grupos avaliados, quanto ao comprimento da perna, contrasta-se com o resultado de SIQUEIRA (1983), que verificou diferença a favor dos mestiços, ao estudar cordeiros da raça Ideal e Texel x Ideal.

Os índices de compacidade, apresentados na Tabela 2, confirmam a inexistência de diferenças significativas entre os grupos genéticos considerados. OSÓRIO (1992), entretanto, obteve diferenças entre os grupos que estudou, tanto para compacidade da carcaça, como da perna. Contudo, é conveniente salientar que esse autor analisou efeito de procedência, trabalhando com carcaças de diferentes idades, desde Merino Alemão (70-80 dias) a carcaças neozeolandesas (5-6 meses de idade).

Os índices de compacidade poderiam representar alternativa para se avaliar objetivamente a conformação das carcaças, considerando-se a relatividade da precisão dos sistemas subjetivos. Entretanto, deve-se considerar dois aspectos: a necessidade de se estudar com mais profundidade e intensidade este tema e a falta de praticidade da implantação deste método em sistemas de classificação de carcaças. De qualquer forma, trata-se de uma metodologia adequada sob o ponto de vista de pesquisa.

As médias e os desvios-padrão para a área de olho de lombo e as medidas realizadas no longissimus dorsi encontram-se na Tabela 3. Verifica-se que não houve diferenças significativas entre os dois tratamentos, para todas essas variáveis, corroborando os resultados SILVEIRA et al. (1980), LOOSE et al. (1981) e SIQUEIRA (1983).

A média geral constatada no presente estudo, 
SIQUEIRA e FERNANDES

Tabela 1 - Médias e desvios-padrão para as medidas da carcaça $(\mathrm{cm})$, nos dois grupos genéticos

Table 1 - Means and standard deviations for carcass measurements (cm), in two genetic groups

Variável Puro Mestiço

Variable Straightbred Crossbred

Comprimento da perna

$30,69^{a^{*}} \pm 0,90(3,0)^{* *} \quad 30,39^{\mathrm{a}} \pm 0,81(3,0)$

Leg length

Comprimento interno da carcaça

$48,75^{\mathrm{a}} \pm 1,30(2,6)$

$47,83^{\mathrm{b}} \pm 1,35(2,8)$

Carcass internal length

Comprimento externo da carcaça

$57,39^{\mathrm{a}} \pm 2,48(4,3)$

$55,42^{\mathrm{b}} \pm 1,84(3,3)$

Carcass external length

Largura da garupa

$14,38^{\mathrm{a}} \pm 0,56(3,8) \quad 14,46^{\mathrm{a}} \pm 0,74(5,0)$

Hindquarters width

Largura da costela

$20,42^{\mathrm{a}} \pm 1,74(8,5)$

$20,98^{\mathrm{a}} \pm 1,79(8,5)$

Ribs rack width

Profundidade do tórax

$24,31^{\mathrm{a}} \pm 1,84(7,5)$

$23,84^{\mathrm{a}} \pm 0,67(2,8)$

Thorax depth

Perímetro da garupa

Hindquarters perimeter

$49,47^{\mathrm{a}} \pm 1,40(2,8)$

$49,47^{\mathrm{a}} \pm 2,33(4,7)$

* Médias, na linha, seguidas de letras diferentes são diferentes $(P<0,05)$ pelo teste Tukey.

** Os valores entre parênteses referem-se aos coeficientes de variação.

* Means, within a row, followed by different letters are different $(P<.05)$ by Tukey test.

** Numbers within parenthesis represent the coefficients of variation.

Tabela 2 - Médias e desvios-padrão para os índices de compacidade dos dois grupos genéticos

Table 2 - Means and standard deviations for compacity index in two genetic groups

\begin{tabular}{lcc}
\hline Variável & Puro & Mestiço \\
Variable & Straightbred & Crossbred \\
\hline $\begin{array}{l}\text { Índice de compacidade da carcaça } \\
\text { Carcass compacity index }\end{array}$ & $0,24^{\mathrm{a} *} \pm 0,01(4,0)^{* *}$ & $0,25^{\mathrm{a}} \pm 0,02(8,0)$ \\
Índice de compacidade da perna & $0,47^{\mathrm{a}} \pm 0,03(6,4)$ & $0,47^{\mathrm{a}} \pm 0,03(6,4)$
\end{tabular}

Leg compacity index

* Médias, na linha, seguidas de letras diferentes são diferentes $(P<0,05)$ pelo teste Tukey.

** Os valores entre parênteses referem-se aos coeficientes de variação.

* Means, within a row, followed by different letters are different $(P<.05)$ by Tukey test.

** Numbers within parenthesis represent the coefficients of variation.

Tabela 3 - Médias e desvios-padrão das medidas A, B $(\mathrm{cm}), \mathrm{C}, \mathrm{J}(\mathrm{mm})$ e área de olho de lombo $\left(\mathrm{cm}^{2}\right)$, nos dois grupos genéticos

Table 3 - Means and standard deviations of measurements $A, B(\mathrm{~cm}), C, J(\mathrm{~mm})$ and loin eye area $\left(\mathrm{cm}^{2}\right)$ in two genetic groups

\begin{tabular}{lcc}
\hline $\begin{array}{l}\text { Variável } \\
\text { Variable }\end{array}$ & $\begin{array}{c}\text { Puro } \\
\text { Straightbred }\end{array}$ & $\begin{array}{c}\text { Mestiço } \\
\text { Crossbred }\end{array}$ \\
\hline $\begin{array}{l}\text { Medida A } \\
\text { Measurement A }\end{array}$ & $5,10^{\mathrm{a}^{*} \pm 0,27(5,3)^{* *}}$ & $5,36^{\mathrm{a}} \pm 0,50(9,3)$ \\
$\begin{array}{l}\text { Medida B } \\
\text { Measurement B }\end{array}$ & $2,41^{\mathrm{a}} \pm 0,31(12,8)$ & $2,49^{\mathrm{a}} \pm 0,32(12,8)$ \\
$\begin{array}{l}\text { Medida C } \\
\text { Measurement } C\end{array}$ & $1,43^{\mathrm{a}} \pm 0,60(42,0)$ & $1,45^{\mathrm{a}} \pm 0,77(53,0)$ \\
$\begin{array}{l}\text { Medida J } \\
\text { Measurement } J\end{array}$ & $3,27^{\mathrm{a}} \pm 1,48(45,2)$ & $3,24^{\mathrm{a}} \pm 1,53(47,2)$ \\
Área de olho de lombo & $8,51^{\mathrm{a}} \pm 1,34(15,7)$ & $9,44^{\mathrm{a}} \pm 2,02(21,4)$
\end{tabular}

Loin eye area

* Médias, na linha, seguidas de letras diferentes são diferentes $(P<0,05)$ pelo teste Tukey.

** Os valores entre parênteses referem-se aos coeficientes de variação.

* Means, within a row, followed by different letters are different $(P<.05)$ by Tukey test.

${ }^{* *}$ Numbers within parenthesis represent the coefficients of variation. 
para a área do longissimus dorsi, está de acordo com os resultados relatados por KIRTON et al. (1972) e BOYLAN et al. (1976), com pesos de abate similares, permitindo afirmar que os cordeiros puros Corriedale apresentaram quantidade de músculo na carcaça semelhante aos mestiços Ile de France x Corriedale.

Outra medida objetiva, que indica a musculatura total da carcaça, é a profundidade do músculo longissimus dorsi (medida B), conforme salientou PALSSON (1939). Todavia, não se constataram, no presente experimento, diferenças significativas entre os tratamentos. Este resultado confirma, mais uma vez, a similaridade dos grupos, quanto à proporção de massas musculares.

Os valores da espessura de gordura (medida C) foram similares entre os grupos genéticos, discordando de PILAR et al. (1993), que obtiveram maior espessura de gordura em cordeiros Corriedale (2,7 $\mathrm{mm}$ ), quando comparados com mestiços Ile de France $\mathrm{x}$ Corriedale $(1,2 \mathrm{~mm})$, abatidos na mesma faixa de peso do presente estudo.

Ainda na Tabela 3, observam-se os valores da medida J, a qual, apesar de não ser referenciada na literatura com frequiência, é uma medida importante, que permite avaliar melhor o perfil da distribuição da gordura de cobertura, tendo em vista que é tomada no ponto em que a mesma se apresente com maior espessura.

Sob o ponto de vista estatístico, mais uma vez, verificou-se que os genótipos não diferiram.

Como demonstram os resultados da Tabela 4, os grupos experimentais apresentaram condições corporais significativamente distintas, sendo os mestiços Ile de France $\mathrm{x}$ Corriedale superiores aos puros Corriedale. Esperar-se-iam condições corporais similares, já que é um reflexo do estado do animal, e não da conformação. Entretanto, tratando-se de uma avaliação subjetiva, pode-se inferir que a conforma- ção, levemente superior nos mestiços, provavelmente influenciou os resultados obtidos.

Os valores constatados para a conformação apresentaram-se, sob o ponto de vista estatístico, diferentes entre os genótipos estudados. A diferença de 0,24 em termos absolutos, apesar de não ter sido muito grande, parece compatível com o grau de sangue dos cordeiros mestiços. Entretanto, espera-se sempre que o uso de raça especializada para a produção de carne, como a Ile de France, a qual apresenta conformação bastante avantajada diante da raça Corriedale, proporcione nos cordeiros mestiços carcaças muito superiores aos puros. De acordo com CAÑEQUE et al. (1989), há poucas diferenças entre raças que possam influir marcadamente na conformação, a ponto de alterar a proporção de músculo total.

Deve-se ressaltar, também, a importância da gordura e de sua localização, como fatores que podem influir na conformação. Neste estudo, porém, observou-se que as carcaças consideradas de melhor conformação (IF x C) apresentaram a mesma quantidade de gordura de cobertura (medida $\mathrm{C}$ - Tabela 3 ) que as carcaças de inferior conformação.

OSÓRIO (1992) relata que as carcaças de melhor conformação subjetiva são as mais curtas, de menor comprimento da perna e, conseqüentemente, de maior compacidade de perna. Os resultados da presente pesquisa, no entanto, são discordantes, em que apenas o comprimento da carcaça foi diferente (Tabela 1) entre as grupos de melhor e pior conformação. Todavia, os coeficientes de correlação da conformação com o comprimento interno da carcaça e comprimento da perna foram $-0,35$ e $-0,38$, respectivamente.

Normalmente, existe no consumidor europeu aversão pelo excesso de gordura, em todos os alimentos, inclusive na carne ovina; já em países árabes, por exemplo, a gordura da carcaça constitui fator impor-

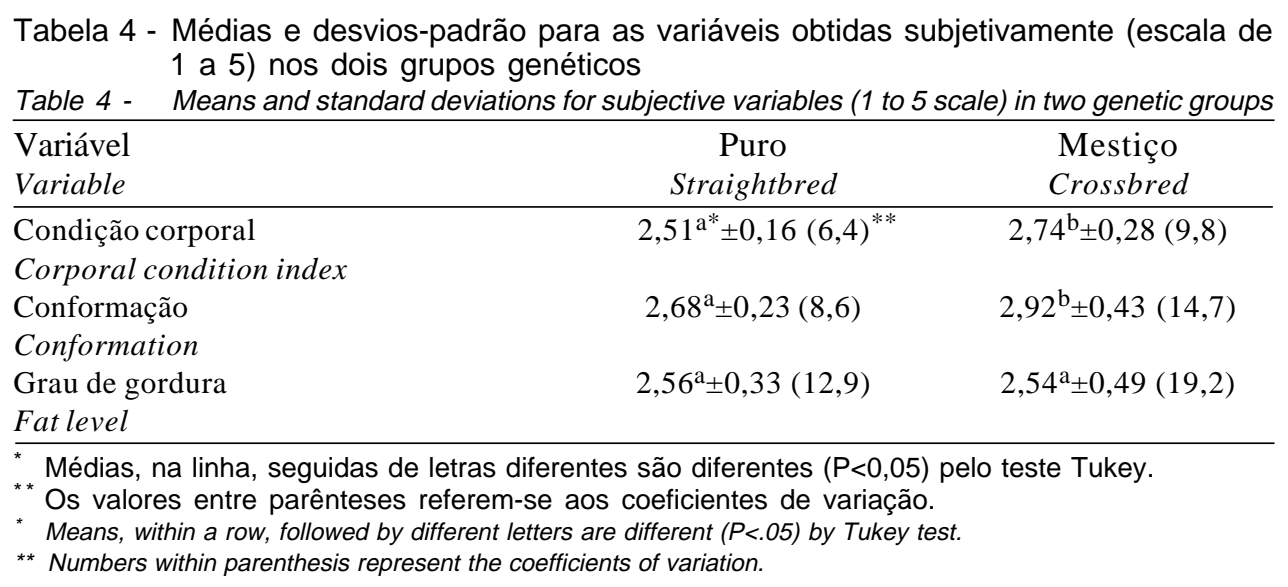


tante de qualidade. A tendência atual é produzir carcaças magras, porém com ótimo grau de gordura, que permita boa apresentação e características sensoriais satisfatórias.

As carcaças deste experimento foram consideradas por intermédio de suas notas, como de gordura mediana, representada por uma capa uniforme, e nãoexcessiva. De acordo com a maioria dos autores consultados, para o mesmo peso vivo e de carcaça, o grau de gordura é função da raça, do sexo e tipo de alimentação.

Verificou-se, quanto ao grau de gordura, que não houve diferença significativa entre os grupos genéticos, discordando dos resultados de THOMPSON e BUTTERFIELD (1987). Entretanto, KIRTON et al. (1967) relatam que, quando as comparações são efetuadas em igual estado de maturidade, a maioria das diferenças entre raças desaparece, corroborando, assim, os resultados obtidos neste experimento.

\section{Conclusões}

Os cordeiros puros e mestiços proporcionaram, de maneira geral, características de carcaça muito similares.

Ambos os genótipos, por um lado, produziram carcaças sem excesso de gordura, por outro atenderam a necessidade de pequena cobertura de gordura, para que a qualidade da carne não seja prejudicada.

Os resultados obtidos não invalidam per si a utilização do cruzamento Ile de France x Corriedale em circunstâncias semelhantes, uma vez que outras variáveis envolvidas com a eficiência global do sistema de produção não foram estudadas.

\section{Referências Bibliográficas}

AKÇAPINAR, H. 1976. Crossbreeding of Turkish Merino breeds with Ile de France. Lalaham Zootekmi Arastirma Enstitüsü. Anim. Breed. Abstr., 44:68 (Abstract).

BOYLAN, W.J., BERGER, Y.M., ALLEN, C.E. 1976. Carcass merit of Finnsheep crossbred lambs. J. Anim. Sci., 42:1413-20.

BONIFACINO, L., KREMER, R., ORLANDO, D. et al. 1979. Estudio comparativo de corderos Corriedale y Corriedale por Texel. 2. Pesos al nascer, ganancias diarias y caracteristicas de la carcasa. Rev. Vet., 70:63-71.

CAÑEQUE, V., HUILDOBRO, F.R., DOLZ, J.F. et al. 1989. Producción de carne de cordero. Madrid: Ministerio de Agricultura Pesca y Alimentacion. 520p.

CARVALHO, J.B.P., PEDROSO, J.R., FIGUEIRÓ, P.R.P. 1980. Alguns fatores que afetam o rendimento de carne ovina. Rev. Cent. Ciênc. Rurais, 10(2):95-104.

COLOMER-ROCHER, F. Estudio de los parametros que definem los caracteres cuantitativos y cualitativos de las canales. In: CURSO INTERNACIONAL SOBRE PRODUCCIÓN DE CARNE Y LECHE CON BASES EN PASTOS Y FORRAGES. La Coruña. Anais... La Coruña, España: 1988. 108p.

EL KARIN, A.I.A., OWEN, J.B., WHITAKER, C.J. 1988.
Measurement on slaughter weight, side weight, carcass joints and their association with carcass composition of two types of Sudan Desert sheep. J. Agric. Sci.,110:65-9.

HUIDOBRO, F., JURADO, I.J. 1989. Meat production of crosbred Manchega sheep. Invest. Agrar. Prod. Sanid. Anim., 4(1):35-44.

KIRTON, A.H., CLARKE, J.N., CARTER, A.H. 1967. Effect of preslaughter fasting or liveweight, carcass weight, and carcass composition of Southdown ram lambs. N.Z.J.Agric. Res., 10:45-55.

KIRTON, A.H., FOURIE, P.D., JURY, K.E. 1972. Growth and development of sheep. 3. Growth of carcass and non carcass components of Southdown and Romney and their cross and some relationships with composition. N. Z. Agric. Res., 10:214-27.

LAMBUTH, T.R.J., KEMP, J.D., GLIMP, H.A. 1970. Effect of rate of gain and slaughter weight on lamb carcass composition. J. Anim. Sci., 30:27-31.

LOOSE, E.M., JARDIM, P.O.C., OSÓRIO, J.C.S. et al. Estudo comparativo de carcaças de cordeiros Ideal com cruzas Ideal $x$ Texel. In: REUNIẨO ANUAL DA SOCIEDADE BRASILEIRA DE ZOOTECNIA, 18, 1981, Goiânia. Anais... Goiânia: SBZ, 1981, p.396.

OSÓRIO, J.C.S. Estudio de la calidad de canales comercializadas en el tipo ternasco segun la procedencia: bases para la mejora de dicha calidad en Brasil. Zaragoza, 1992.335p. Tese (Doutorado em Veterinaria) - Facultad de Veterinaria, Universidad de Zaragoza, 1992.

PALSSON, H. 1939. Meat qualities in sheep with special reference to Scottish breed and sample joint as indice of quality and composition. J. Agric. Sci., 29:544-625.

PILAR, R.C., CASSOL, C., RESTLE, J. et al. 1993. Desempenho e características de carcaças ovinas confinadas. Inf. Cerro Coroado, 5:8-9.

SAÑUDO, C., SIERRA, I. 1986. Calidad de la canal en la especie ovina. Ovino, (1):127-53.

SIERRA, I., BOCCARD, R. La armonia anatômica en el ganado ovino. In: SYMPOSIUM SOBRE LOS PROBLEMAS DE LA PRODUCCION OVINA BAJO CONDICIONES DE MEDIO DIFICILES, 1, 1970, Zaragoza. Proccedings ... Zaragoza: 1970. p.495.

SIERRA, I., SAÑUDO, C., ALCALDE, M.J. 1992. Calidad de la canal en corderos ligeros tipo ternasco: canales españolas y de importación. Inf. Téc. Econ. Agrár., 88(1):88-94.

SILVEIRA, P.M., OSÓRIO, J.C.S., JARDIM, P.O.C. et al. Efeito da raça sobre o peso vivo e características da carcaça em ovinos. In: REUNIÃO ANUAL DA SOCIEDADE BRASILEIRA DE ZOOTECNIA, 17, 1980, Fortaleza. Anais... Fortaleza: SBZ, 1980. p.112.

SIQUEIRA, E.R. Desempenho e características de carcaças de corderos machos e fêmeas da raça Ideal e cruzas Texel x Ideal, criados em pastagem nativa. Pelotas: UFPel, 1983. 130p. Dissertação (Mestrado em Produção Animal) - Universidade Federal de Pelotas, 1983.

THOMPSON, J.M., BUTTERFIELD, R.M. 1987. Food intake, growth and body composition in Australian Merino sheep selected for high and low weaning weight. 4. Partitioning of dissected and chemical fat in the body. Anim. Prod., 45:49-60.

THORNTON, R.F., SHAW, F.D., HOOD, R.L. 1974. Marbling in feedlot cattle. Aust. J. Anim. Husb, 14:281-5.

THWAITES, C.J., YEATES, N.T.M., POGUE, R.F. 1964. Objective appraisal of intact lamb and mutton carcasses. $J$. Agric. Sci., 63:415-20.

ZAR, J.H. 1984. Biostatistical analysis 2.ed. Englewood Cliffs: Prentice Hall. 718p.

Recebido em: 09/02/99 Aceito em: 16/06/99 